\title{
Convex Quantum Logic
}

\author{
Federico Holik ${ }^{1}$, Cesar Massri ${ }^{2}$ and Nicolás Ciancaglini ${ }^{1}$
}

March 12, 2018

\author{
1- Instituto de Astronomía y Física del Espacio (IAFE) \\ Casilla de Correo 67, Sucursal 28, 1428 Buenos Aires, Argentina \\ 2- Departamento de Matemática - Facultad de Ciencias Exactas y Naturales \\ Universidad de Buenos Aires - Pabellón I, Ciudad Universitaria \\ Buenos Aires, Argentina
}

\begin{abstract}
In this work we study the convex set of quantum states from a quantum logical point of view. We consider an algebraic structure based on the convex subsets of this set. The relationship of this algebraic structure with the lattice of propositions of quantum logic is shown. This new structure is suitable for the study of compound systems and shows new differences between quantum and classical mechanics. This differences are linked to the nontrivial correlations which appear when quantum systems interact. They are reflected in the new propositional structure, and do not have a classical analogue. This approach is also suitable for an algebraic characterization of entanglement.
\end{abstract}

Key words: entanglement-quantum logic-convex sets

\section{Introduction}

There exists a widely discussed distinction between proper and improper mixtures [1, 2]. In this work we adopt this distinction as a starting point. From this point of view, improper mixtures do not admit an ignorance interpretation, as is the case for proper mixtures (for more discussion on this subject see also [3, 44 and [5]). One of the most important consequences of this fact is that improper mixtures have to be considered as states on their own right, besides pure states.

Furthermore, the study of the convex set of quantum states plays a central role in decoherence 6] and quantum information processing [7, and this convex set is formed mainly by mixed states. For a Hilbert space of finite dimension $N$, pure states form a $2(N-1)$-dimensional subset of the $\left(N^{2}-2\right)$-dimensional boundary of the convex set of states. Thus, regions of the convex set of states are the important objects to investigate, rather than the lattice of projections.

The standard quantum logical approach to quantum mechanics $(Q M)[8],[9],[10],[11$, takes the lattice of projections of the Hilbert space of the system to be the lattice of propositions (see section 2 for a brief review). In this framework, as in classical mechanics $(C M)$, the state of the system is in direct correspondence with the conjunction of all its actual properties. This 
conjunction yields a pure state or a ray in the Hilbert space, which corresponds to an atom of the lattice of projections. But when we consider the system $S$ formed by subsystems $S_{1}$ and $S_{2}$, the following problem appears (see for example [12]). If $S$ is in an entangled (pure) state, the sates of the subsystems become improper mixtures, and so, it is no longer true that the conjunction of all actual properties yields the true state of the subsystem. On the contrary, the conjunction yields a non atomic proposition of the von Newmann lattice of projections $\left(\mathcal{L}_{v \mathcal{N}}\right)$, i.e., a subspace of dimension greater than one [13, and leaves the state undetermined. Thus, this procedure does not give the real physical state of the system any more. We analyze this problem in detail in section 3, where we review and extend the discussion posed in [14], and impose general conditions for the structures that we want to construct.

The usual way to incorporate improper mixtures (or more generally, states) in the $Q L$ approach, is as measures over $\mathcal{L}_{v \mathcal{N}}$ (or equivalently, measures over the propositions of the abstract lattice). So, in the study of cases which may involve improper mixtures, we have to jump to a different level than that of the propositions (projections). The physical propositions belong to $\mathcal{L}_{v \mathcal{N}}$, while mixtures belong to the set of measures over $\mathcal{L}_{v \mathcal{N}}$. We claim that this split is at the heart of the problem posed in 12, and complementarily, in section 3 of this article. We will review this in detail in sections 2 and 3 .

In section 3 we give a list of conditions for the structures that we are looking for, in order to grant that they solve the posed problems. We work out a structure which incorporates improper mixtures in the same status as pure states (alike $\mathcal{L}_{v \mathcal{N}}$ ), i.e., in which all states of the system are atoms of the lattice. A first approach in this direction was done in [14], where an extension of the von Newmann lattice of projections was built. In that article it was shown that it is possible to construct a lattice theoretical framework which incorporates improper mixtures as atoms, denoted by $\mathcal{L}$ here. We briefly reproduce (without proof), some of the results of [14] in section 4 .

In this work we extend the construction of 14 to a larger structure $\mathcal{L}_{\mathcal{C}}$, formed by all convex subsets of $\mathcal{C}$. This is developed in sections 5 and [6. As desired, this extension solves the problem possed in section 3. We think that this approach is suitable in order to consider decoherence or entangled systems from a quantum logical and algebraic point of view. Furthermore, taking the convex set of states as an starting point could be of interest if we take into account that there exists a formulation of $Q M$ in terms of convex sets (see [15], [16] and [17]). This is an independent formulation of $Q M$ and has the advantage that it can include models of theories which cannot be represented by Hilbert spaces, as is the case of non linear generalizations of quantum mechanics.

Using $\mathcal{L}_{\mathcal{C}}$, we can construct projection functions from the lattice of the whole system to the lattices of the subsystems which satisfy, in turn, to be compatible with the physical description. A similar construction can be made for $\mathcal{L}$ (see section [4). Alike the von Newmann case, where these projection functions do not exist, the projections defined in $\mathcal{L}$ and $\mathcal{L}_{\mathcal{C}}$ satisfy this condition. They are also canonical in the sense that they are constructed using partial traces, in accordance with the quantum formalism.

The approach presented here shows (as well as the one presented in [14]), the radical difference between quantum mechanics and classical mechanics when two systems interact, a difference which is not properly expressed in the orthodox $Q L$ approach. When dealing with classical systems, no enlargement of the lattice of propositions is needed even in the presence of interactions. The phase space is sufficient in order to describe all relevant physics about the subsystems. But the existence of non-trivial correlations in quantum mechanics forces an enlargement of the state space of pure states to the convex set $\mathcal{C}$, and so, the enlargement of $\mathcal{L}_{v \mathcal{N}}$ to a structure as $\mathcal{L}$ or $\mathcal{L}_{\mathcal{C}}$ (or any other structure which satisfies conditions listed in section 3 ).

In section 6.3 we study the maps between $\mathcal{L}_{\mathcal{C}}, \mathcal{L}_{\mathcal{C}_{1}}$ and $\mathcal{L}_{\mathcal{C}_{2}}$ (the lattices of $S$ and its subsystems), and show that our construction allows for an algebraic characterization of entanglement, showing a new feature which is not so explicit in the standard $Q L$ approach. Finally, in section 
7 we expose our conclusions.

\section{The Convex Set of States and Improper Mixtures}

Let us review first the quantum logical approach to the description of physical systems (see for example [9]). In the standard $Q L$ approach, properties (or propositions) of a quantum system are in correspondence with closed subspaces of a Hilbert space $\mathcal{H}$. The set of subspaces $\mathcal{P}(\mathcal{H})$ with the partial order defined by set inclusion $\subseteq$, intersection of subspaces $\cap$ as the lattice meet, closed linear spam of subspaces $\oplus$ as the lattice join and orthocomplementation $\perp$ as lattice complement, gives rise to an orthomodular lattice $\mathcal{L}_{v \mathcal{N}}(\mathcal{H})=<\mathcal{P}(\mathcal{H}), \cap, \oplus, \neg, 0,1>$ where 0 is the empty set $\emptyset$ and 1 is the total space $\mathcal{H}$. This is the Hilbert lattice, named $Q L$ by Birkhoff and von Neumann. We will refer to this lattice as $\mathcal{L}_{v N}$, the 'von Neumann lattice' (or simply $\mathcal{L}(\mathcal{H}))$.

Mixed states represented by density operators had a secondary role in the classical treatise by von Newmann because they did not add new conceptual features to pure states. In fact, in his book, mixtures meant "statistical mixtures" of pure states [18, pg. 328], which are known in the literature as "proper mixtures" [1, Ch. 6]. They usually represent the states of realistic physical systems whose preparation is not well described by pure states. In the standard formulation of $Q L$, mixtures (as well as pure states) are included as measures over the lattice of projections 19, that is, a state $s$ is a function:

$$
s: \mathcal{L}(\mathcal{H}) \longrightarrow[0 ; 1]
$$

such that:

1. $s(\mathbf{0})=0$ ( $\mathbf{0}$ is the null subspace).

2. For any pairwise orthogonal family of projections $P_{j}, s\left(\sum_{j} P_{j}\right)=\sum_{j} s\left(P_{j}\right)$

But while pure states can be put in a bijective correspondence to the atoms of $\mathcal{L}(\mathcal{H})$, this is not the case for mixtures. We review in Section 3 how this difference leads to problems when compound systems are considered. We must pay attention to improper mixtures ([1, [2]) because we have to deal with them in each (non trivial) case in which a part of the system is considered.

For a classical system with phase space $\Gamma$, the lattice of propositions is defined as the the set of subsets of $\Gamma(\mathcal{P}(\Gamma))$, endowed with set intersection as conjunction " $\wedge$ ", set union as disjunction "У" and set complement as negation " $\neg$ ". We will call this lattice $\mathcal{L}_{\mathcal{C} \mathcal{M}}$. The points $(p, q) \in \Gamma$ are in a bijective correspondence with the states of the system. Statistical mixtures are represented as measurable functions:

$$
\sigma: \Gamma \longrightarrow[0 ; 1]
$$

such that $\int_{\Gamma} \sigma(p, q) d^{3} p d^{3} q=1$.

For quantum compound systems $S_{1}$ and $S_{2}$, given the Hilbert state spaces $\mathcal{H}_{1}$ and $\mathcal{H}_{2}$ as representatives of two systems, the pure states of the compound system are given by rays in the tensor product space $\mathcal{H}=\mathcal{H}_{1} \otimes \mathcal{H}_{2}$. It is not true that any pure state of the compound system factorizes after the interaction in pure states of the subsystems. This situation is very different from that of classical mechanics, where for state spaces $\Gamma_{1}$ and $\Gamma_{2}$, we assign $\Gamma=\Gamma_{1} \times \Gamma_{2}$ for the compound system.

Let us now briefly review the relationship between the states of the joint system and the states of the subsystems in the quantum mechanical case. Let us focus for simplicity on the case 
of two systems, $S_{1}$ and $S_{2}$. If $\left\{\left|x_{i}^{(1)}\right\rangle\right\}$ and $\left\{\left|x_{i}^{(2)}\right\rangle\right\}$ are the corresponding orthonormal basis of $\mathcal{H}_{1}$ and $\mathcal{H}_{1}$ respectively, then the set $\left\{\left|x_{i}^{(1)}\right\rangle \otimes\left|x_{j}^{(2)}\right\rangle\right\}$ forms an orthonormal basis for $\mathcal{H}_{1} \otimes \mathcal{H}_{2}$. A general (pure) state of the composite system can be written as:

$$
\rho=|\psi\rangle\langle\psi|
$$

where $|\psi\rangle=\sum_{i, j} \alpha_{i j}\left|x_{i}^{(1)}\right\rangle \otimes\left|x_{j}^{(2)}\right\rangle$. And if $M$ represents an observable, its mean value $\langle M\rangle$ is given by:

$$
\operatorname{tr}(\rho M)=\langle M\rangle
$$

If observables of the form $O_{1} \otimes \mathbf{1}_{2}$ and $\mathbf{1}_{1} \otimes O_{2}$ (with $\mathbf{1}_{1}$ and $\mathbf{1}_{2}$ the identity operators over $\mathcal{H}_{1}$ and $\mathcal{H}_{2}$ respectively) are considered, then partial state operators $\rho_{1}$ and $\rho_{2}$ can be defined for systems $S_{1}$ and $S_{2}$. The relation between $\rho, \rho_{1}$ and $\rho_{2}$ is given by:

$$
\rho_{1}=\operatorname{tr}_{2}(\rho) \quad \rho_{2}=\operatorname{tr}_{1}(\rho)
$$

where $t r_{i}$ stands for the partial trace over the $i$ degrees of freedom. It can be shown that:

$$
\operatorname{tr}_{1}\left(\rho_{1} O_{1} \otimes \mathbf{1}_{2}\right)=\left\langle O_{1}\right\rangle
$$

and that a similar equation holds for $S_{2}$. Operators of the form $O_{1} \otimes \mathbf{1}_{2}$ and $\mathbf{1}_{1} \otimes O_{2}$ represent magnitudes related to $S_{1}$ and $S_{2}$ respectively. When $S$ is in a product state $\left|\varphi_{1}\right\rangle \otimes\left|\varphi_{2}\right\rangle$, the mean value of the product operator $O_{1} \otimes O_{2}$ will yield:

$$
\operatorname{tr}\left(\left|\varphi_{1}\right\rangle \otimes\left|\varphi_{2}\right\rangle\left\langle\varphi_{1}\right| \otimes\left\langle\varphi_{2}\right| O_{1} \otimes O_{2}\right)=\left\langle O_{1}\right\rangle\left\langle O_{2}\right\rangle
$$

reproducing statistical independence.

Mixtures are represented by positive, Hermitian and trace one operators, (also called 'density matrices'). The set of all density matrixes forms a convex set (of states), which we will denote by $\mathcal{C}$. Remember that the physical observables are represented by elements of $\mathcal{A}$, the $\mathbb{R}$-vector space of Hermitian operators acting on $\mathcal{H}$ :

Definition 2.1. $\mathcal{A}:=\left\{A \in B(\mathcal{H}) \mid A=A^{\dagger}\right\}$

Definition 2.2. $\mathcal{C}:=\{\rho \in \mathcal{A} \mid \operatorname{tr}(\rho)=1, \rho \geq 0\}$

where $B(\mathcal{H})$ stands for the algebra of bounded operators in $\mathcal{H}$. The set of pure states satisfies:

$$
P:=\left\{\rho \in \mathcal{C} \mid \rho^{2}=\rho\right\}
$$

This set is in correspondence with the rays of $\mathcal{H}$ by the association:

$$
\mathcal{F}: \mathbb{C P}(\mathcal{H}) \longmapsto \mathcal{C} \quad \mid \quad[|\psi\rangle] \longmapsto|\psi\rangle\langle\psi|
$$

where $\mathbb{C P}(\mathcal{H})$ is the projective space of $\mathcal{H}$, and $[|\psi\rangle]$ is the class defined by the vector $|\psi\rangle$ $(|\varphi\rangle \sim|\psi\rangle \longleftrightarrow|\varphi\rangle=\lambda|\psi\rangle, \lambda \neq 0)$. $\mathcal{C}$ is a convex set inside the hyperplane $\{\rho \in \mathcal{A} \mid \operatorname{tr}(\rho)=1\}$ formed by the intersection of this hyperplane with the cone of positive matrixes.

Separable states are defined [20] as those states of $\mathcal{C}$ which can be written as a convex combination of product states:

$$
\rho_{\text {Sep }}=\sum_{i, j} \lambda_{i j} \rho_{i}^{(1)} \otimes \rho_{j}^{(2)}
$$


where $\rho_{i}^{(1)} \in \mathcal{C}_{1}$ and $\rho_{j}^{(2)} \in \mathcal{C}_{2}, \sum_{i, j} \lambda_{i j}=1$ and $\lambda_{i j} \geq 0$. So, the set $\mathcal{S}(\mathcal{H})$ of separable states is defined as:

Definition 2.3. $\mathcal{S}(\mathcal{H}):=\{\rho \in \mathcal{C} \mid \rho$ is separable $\}$

As said above, it is a remarkable fact that there are many states in $\mathcal{C}$ which are non separable. If the state is non-separable, it is said to be entangled [21. The estimation of the volume of $\mathcal{S}(\mathcal{H})$ is of great interest (see -among others- 22], 7] and [23]).

In classical mechanics mixtures are not of a fundamental nature. They represent an state of ignorance of the observer, because we know in principle that the system is in a given state $s$ of phase space. On he other hand, in quantum mechanics, we must take into account the difference between proper and improper mixtures. A proper mixture can be considered as a density matrix plus a piece of classical information, which encodes classical probabilities for preparations of ensembles of pure states. This extra piece of classical information may have its source on imperfections of the preparation procedure, or could be produced deliberately, but the important fact is that these probabilities could be determined -at least- in principle, as is the case of mixtures in classical mechanics. But for the case of improper mixtures, this information does not exist in the world.

What is one of the main implications of the fact that improper mixtures do not admit an ignorance interpretation? For the standard formulation of $Q M$ we have at hand what it is usually called "the superposition principle":

Principle 2.4. Superposition Principle. If $\left|\psi_{1}\right\rangle$ and $\left|\psi_{1}\right\rangle$ are physical states, then $\alpha\left|\psi_{1}\right\rangle+\beta\left|\psi_{1}\right\rangle$ $\left(|\alpha|^{2}+|\beta|^{2}=1\right)$ will be a physical state too.

Are there other operations which allows us to form new states up from two given states? If we accept that improper mixtures are states of a fundamental nature as much as pure states do, then, the fact that we can create new physical states mixing two given states, could be thought as a principle which stands besides the superposition principle:

Principle 2.5. Mixing Principle. If $\rho$ and $\rho^{\prime}$ are physical states, then $\alpha \rho+\beta \rho^{\prime}(\alpha+\beta=1$, $\alpha, \beta \geq 0$ ) will be a physical state too.

Mixing principle is not contained directly in the superposition principle. Mixing principle appears as a consequence of the axiom which states that to a compound system corresponds the tensor product of Hilbert spaces. It expresses the fact that improper mixtures are physical states. We will not consider proper mixtures in this work, we only concentrate in physical states.

There is a remarkable physical consequence of all this (which we think is not properly emphasized in the literature). While for pure states there always exist "true propositions" [11], i.e., propositions for which a test will yield the answer "yes" with certainty (and a similar situation for "false propositions"), the situation is radically different for improper mixtures. If we accept that improper mixtures are states of a fundamental nature as well as pure states, then we must face the fact that there exist states for which no "true propositions" exist (discarding the trivial proposition represented by the Hilbert space itself). This is the case for example for the maximum uncertainty state (finite dimension), $\rho=\frac{1}{N} \mathbf{1}$.

\section{The Limits of $\mathcal{L}_{v \mathcal{N}}$}

In the standard $Q L$ approach there is a bijective correspondence between the atoms of the lattice $\mathcal{L}_{v \mathcal{N}}$ and pure states. Each pure state $s$, which is represented by a ray $[|\psi\rangle]$, is an atom of the lattice of projections $\mathcal{L}_{v \mathcal{N}}$. The relationship between the atoms of the lattice and actual properties $p$ of the system (represented by closed subspaces of $\mathcal{H}$ ) is given by: 


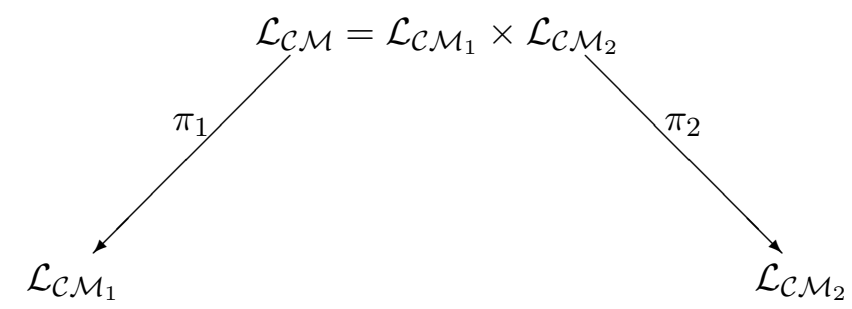

Figure 1: In the classical case, we can map a state of the system to the states of the subsystems using the set-theoretical projections $\pi_{1}$ and $\pi_{2}$

$$
\{s\}=\wedge\left\{p \in \mathcal{L}_{v \mathcal{N}} \mid p \text { is actual }\right\}
$$

and a similar relation holds in the classical case. But there appears a subtle problem with equation 3.0.11 when compound systems are considered. Suppose that $S_{1}$ and $S_{2}$ are subsystems of a larger system $S$ in a pure entangled state $|\psi\rangle$. What happens if we want to determine the states of its subsystems using equation 3.0.11? This problem is studied in 12. If we make the conjunction of all actual properties for, say $S_{1}$, we will no longer obtain an atom of $\mathcal{L}_{v \mathcal{N}_{1}}$. Instead of it, we will obtain a property which corresponds to a subspace of dimension greater than one (see theorem 18 of [13]). But this property does no longer corresponds to the actual state of the subsystem. The state of the subsystem is not a pure state, but an improper mixture given by the partial trace $\operatorname{tr}_{2}(|\psi\rangle\langle\psi|)$. So, it is not possible to obtain the actual physical state of $S_{1}$ using the properties of $\mathcal{L}_{v \mathcal{N}_{1}}$ and equation 3.0.11

Consider figures 2 and 3, Alike the classical case, in general, we will not be able to map states from $\mathcal{L}_{v \mathcal{N}}$ into states of $\mathcal{L}_{v \mathcal{N} i}(i=1,2)$ using partial traces (which are the physical maps that we should use). This is because the states of the subsystems are represented by improper mixtures which are not projections, and thus, they do not belong to $\mathcal{L}_{v \mathcal{N} i}(i=1,2)$. How can we complete the "?" symbols of figure 32? There is no way to do that at the lattices level (when we use von Newmann lattices). We should have to jump into the level of measures over $\mathcal{L}_{v \mathcal{N}_{1}}$. But in this work we want to avoid this possibility.

As said above, statistical mixtures of $C M$ do not have a fundamental nature; on the contrary, they represent a state of knowledge of the observer, a loss of information. Alike classical mixtures, improper mixtures are of a fundamental nature; according with the orthodox interpretation of $Q M$, there is no other information available about the state of the subsystem. As well as pure states (and points of classical phase space), they represent pieces of information that are maximal and logically complete. They cannot be consistently extended and they decide any property or the result of any experiment on the given subsystem. They determine the physics of the subsystem. Due to this, we want to consider improper mixtures as states in the same level to that of pure states, i.e., we want that they belong to the same propositional structure.

The strategy that we follow in this work is to search for structures which contain improper mixtures in such a way that they have an equal treatment as the one given to pure states. As we will see, this is possible, and such structures can be defined in a natural way, extending (in a sense explained below) $\mathcal{L}_{v \mathcal{N}}$ and in a way which is compatible with the physics of compound quantum systems.

We want to avoid the fact that actual properties of the propositional system do not determine the state of the system, understood as the state of affairs which determines its physics. We think that every reasonable notion of physical state in a propositional system should satisfy equation 3.0.11.

As remarked above, there are improper mixtures for which all yes-no tests are uncertain. But it is important to remark that this does not imply that the system has no testable properties at all. Making quantum state tomographies we can determine the state of the system. These kind of "tests" however, are of a very different nature than that of the yes-no experiments. But the only thing that we care about is that of the reality of physical process and our capability of 


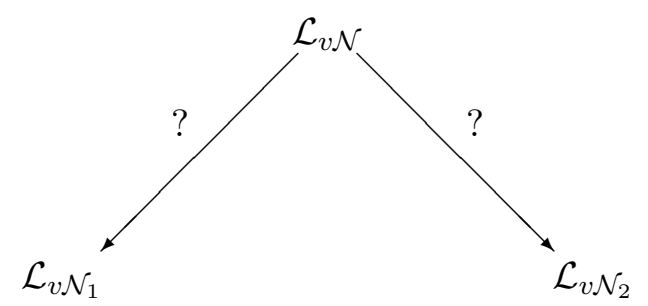

Figure 2: We cannot apply partial traces in order to go down from $\mathcal{L}_{v \mathcal{N}}$ to $\mathcal{L}_{v \mathcal{N}_{1}}$, and $\mathcal{L}_{v \mathcal{N}_{2}}$. How do we complete the "?" symbols?

experimentally test this reality. We search for structures which reflect this physics in a direct way.

There are other reasons for considering structures which contains improper mixtures in a same status as that of pure states. There are a lot of studies of interest which concentrate on mixtures. For example, this is the case in quantum decoherence, quantum information processing, or the independent generalizations of quantum mechanics which emphasize the convex nature of mechanics (not necessarily equivalent to "Hilbertian" $Q M$ ). The set of interest in these studies is $\mathcal{C}$ instead of the lattice of projections. So it seems to be adequate to study structures which include improper mixtures as well as pure states in a same level of "discourse". Such structures could provide a natural framework in which we study foundational issues related to these topics.

Let us see examples of physical situations which could be captured by propositional structures based on $\mathcal{C}$. Suppose that we have a system $S_{1}$ in a given state $\rho_{1}$ (which can be an improper mixture). If we consider its environment $S_{2}$, then we may state the proposition "the state of affairs is such that $S_{1}$ is in state $\rho_{1}$ ". We note that when we look the things from the point of view of the total system $S=$ System + Environment, a convex subset of $\mathcal{C}$ (the convex set of states of $S$ ) corresponds to this proposition. This is so because $S$ can be in any state $\rho$ such that $\operatorname{tr}_{2}(\rho)=\rho_{1}$, and this corresponds to the convex set $\operatorname{tr}_{2}^{-1}\left(\left\{\rho_{1}\right\}\right)$ (see section 6.4). Similarly, we obtain the convex set $\operatorname{tr}_{2}^{-1}\left(\left\{\rho_{1}\right\}\right) \cap \operatorname{tr}_{1}^{-1}\left(\left\{\rho_{2}\right\}\right)$ for the proposition $S_{1}$ is in state $\rho_{1}$ and $S_{2}$ is in state $\rho_{2}$. This propositions represent the ignorance that we have about the actual state of the whole system. A propositional structure which includes propositions of this kind could be useful, and more natural for the study of quantum information.

It is important to notice that propositions such as the one represented by $\operatorname{tr}_{2}^{-1}\left(\left\{\rho_{1}\right\}\right)$ above cannot be tested by yes-no experiments in general. Notwithstanding, they represent actual states of affairs, and they can certainly be tested making measures on correlations, quantum tomographies, etc.

As another example, consider the von Newmann entropy $S(\rho)=-\operatorname{tr}(\rho \ln (\rho))$. It has the following property of concavity [21]

Proposition 3.1. If $\rho=\alpha \rho_{1}+(1-\alpha) \rho_{2}, 0 \leq \alpha \leq 1$, we have

$$
S(\rho) \geq \alpha S\left(\rho_{1}\right)+(1-\alpha) S\left(\rho_{2}\right)
$$

Now consider the proposition "the entropy of the system is greater than $S_{0}$ ". To such a proposition -which has a very definite physical meaning- there corresponds a convex subset of $\mathcal{C}$. This is so, because if we consider the set

$$
S_{\geq S_{0}}=\left\{\rho \in \mathcal{C} \mid S(\rho) \geq S_{0}\right\}
$$

and if $\rho_{1}, \rho_{2} \in S_{\geq S_{0}}$, then any convex combination $\rho=\alpha \rho_{1}+(1-\alpha) \rho_{2}$-due to the concavity property- will also belong to $S_{\geq S_{0}}$. This example shows that there are propositions with a very clear physical meaning which correspond to subsets of $\mathcal{C}$ instead of subspaces of the Hilbert space.

We summarize below the desired properties for the lattices that we are searching for, in order to solve the problems posed in this section: 
1 All physical states are included as atoms of the the new lattice. Atoms and physical states are in one to one correspondence.

2 A state of the system will be the conjunction of all the actual properties (i.e. elements of the structure). This means that actual properties determine univocally the state of the system.

3 There exist projection functions which map all states (atoms) of the structure corresponding to the whole system $S$, to the corresponding states (atoms) of its subsystems $S_{1}$ and $S_{2}$. This assignation rule must be compatible with the physics of the problem.

$4 \mathcal{L}_{v \mathcal{N}}$ is set theoretically included in the new structure, in order to preserve physical properties in the standard sense.

5 Given two propositions of the structure there must exist an operation which yields a proposition which expresses the fact that we can form mixtures of states.

There is a trivial example which satisfies the conditions $1-4$ listed above, namely, the set of all subsets of $\mathcal{C}$, which we denote $\mathcal{P}(\mathcal{C})$. Using set intersection as conjunction and set union as disjunction, it is a boolean lattice. If we fix an entanglement measure, consider the proposition "the system has such amount of entanglement" or given an entropy measure, we can say "the system has such amount of entropy" and so on. To such propositions we can assign elements of $\mathcal{P}(\mathcal{C})$, the set of all states which satisfy those propositions. But the boolean "or" defined by the set union hides the fact that in quantum mechanics we can make superpositions of states (principle 2.4) and that we can mix states (principle 2.5). In this work we search for structures which satisfy condition 5 . For that reason, the lattice formed by $\mathcal{P}(\mathcal{C})$ (from now on $\mathcal{L}_{\mathcal{B}}$ ) is not of our interest. It expresses the almost trivial fact that we can make propositions such as "the states of $\mathcal{C}$ which make a given function to have such a value" but it hides the radical differences between $Q M$ and $C M$.

We can define -at least- two structures which satisfy the above list. One of them which we call $\mathcal{L}$ (see section 4 and [14]) is in close connection with the lattice of subspaces of the space of hermitian matrixes. In this work it plays the role of a technical step to reach $\mathcal{L}_{\mathcal{C}}$ (section [5), the lattice formed by the convex subsets of $\mathcal{C}$. We show below that the study of these structures sheds light on the study of compound quantum systems, and provide a suitable (natural) language for them, mainly because of they sort the problems possed above. They show things that $\mathcal{L}_{v \mathcal{N}}$ hides, or in other words, which are not expressed clearly. For example, given two pure states $\rho_{1}=\left|\psi_{1}\right\rangle\left\langle\psi_{1}\right|$ and $\rho_{2}=\left|\psi_{2}\right\rangle\left\langle\psi_{2}\right|$ we can apply the "or" operation of $\mathcal{L}_{v \mathcal{N}}, \vee_{\mathcal{L}_{v \mathcal{N}}}$, which yields the linear (closed) spam of $\left|\psi_{1}\right\rangle$ and $\left|\psi_{2}\right\rangle$. But we can also consider the " $\vee_{\mathcal{L}_{\mathcal{C}}}$ " operation (see section 5), which yields all statistical mixtures of the form $\alpha \rho_{1}+(1-\alpha) \rho_{2}$. This operation is different from linear combination (quantum superpositions), and is related to the -non classicalmixing of states (improper mixing). This "mixing" operation cannot be represented in $Q L$ at the level of $\mathcal{L}_{v \mathcal{N}}$ itself, i.e., it is not a lattice operation, but it has to be represented at the level of statistical mixtures (measures over $\mathcal{L}_{v \mathcal{N}}$ ).

It is important to notice that it is not the aim of this work to replace the von Newmann lattice by these new structures, but to stress its limitations for the problem of compound systems and to define its domain of applicability. We adopt the point of view that these constructions -including $\mathcal{L}_{v \mathcal{N}^{-}}$yield different complementary views of quantum systems. In the following sections, we present $\mathcal{L}$ and $\mathcal{L}_{\mathcal{C}}$. 


\section{The Lattice $\mathcal{L}$}

In this section we review (without proof) some results and definitions of [14]. Let us define $G(\mathcal{A})$ as the lattice associated to the pair $(\mathcal{A}, \operatorname{tr})$, where $\mathcal{A}$ is considered as an $\mathbb{R}$-vector space and $\operatorname{tr}$ is the usual trace operator on $B(\mathcal{H})$, which induces the scalar product $\langle A, B\rangle=\operatorname{tr}(A \cdot B)$ $(\operatorname{dim}(\mathcal{H})<\infty)$. The restriction to $\mathcal{A}$ of tr, makes $\mathcal{A}$ into an $\mathbb{R}$-euclidean vector space.

$$
G(\mathcal{A}):=\{S \subset \mathcal{A} \mid S \text { is a } \mathbb{R} \text { - subspace }\}
$$

$G(\mathcal{A})$ is a modular, orthocomplemented, atomic and complete lattice (not distributive, hence not a Boolean algebra). Let $\mathcal{L}$ be the induced lattice in $\mathcal{C}$ :

$$
\mathcal{L}:=\{S \cap \mathcal{C} \mid S \in G(\mathcal{A})\}
$$

There are a lot of subspaces $S \in G(\mathcal{A})$ such that $S \cap \mathcal{C}=S^{\prime} \cap \mathcal{C}$, so for each $L \in \mathcal{L}$ we choose as a representative the subspace with the least dimension:

$$
\min \left\{\operatorname{dim}_{\mathbb{R}}(S) \mid L=S \cap \mathcal{C}, S \in G(\mathcal{A})\right\}
$$

Let $[S]=L$, being $S \in G(\mathcal{A})$ an element of the class $L$, then

$$
\begin{aligned}
S \cap \mathcal{C} \subseteq<S \cap \mathcal{C}>_{\mathbb{R}} \subseteq S \Rightarrow & S \cap \mathcal{C} \cap \mathcal{C} \subseteq<S \cap \mathcal{C}>_{\mathbb{R}} \cap \mathcal{C} \subseteq S \cap \mathcal{C} \Rightarrow \\
& <S \cap \mathcal{C}>\cap \mathcal{C}=S \cap \mathcal{C}
\end{aligned}
$$

So $\langle S \cap \mathcal{C}>$ and $S$ are in the same class $L$. Note that $\langle S \cap \mathcal{C}>\subseteq S$ and if $S$ is the subspace with the least dimension, then $\langle S \cap \mathcal{C}\rangle=S$. Also note that the representative with least dimension is unique, because if we choose $S^{\prime}$ such that $S^{\prime} \cap \mathcal{C}=S \cap \mathcal{C}$, then

$$
S=<S \cap \mathcal{C}>=<S^{\prime} \cap \mathcal{C}>=S^{\prime}
$$

Finally, the representative of a class $L$ that we choose is the unique $\mathbb{R}$-subspace $S \subseteq \mathcal{A}$ such that

$$
S=<S \cap \mathcal{C}>\mathbb{R}
$$

We call it the good representative. It is important to remark that in the case of infinite dimensional Hilbert spaces we cannot define good representatives in such a way.

Let us now define " $\vee$ ", " $\wedge$ " and " $\neg$ " operations and a partial ordering relation " $\longrightarrow$ ( or equivalently " $\leq$ ") in $\mathcal{L}$ as:

$$
\begin{gathered}
(S \cap \mathcal{C}) \wedge(T \cap \mathcal{C}):=<S \cap \mathcal{C}>\cap<T \cap \mathcal{C}>\cap \mathcal{C} \\
(S \cap \mathcal{C}) \vee(T \cap \mathcal{C}):=(<S \cap \mathcal{C}>+<T \cap \mathcal{C}>) \cap \mathcal{C} \\
(S \cap \mathcal{C}) \longrightarrow(T \cap \mathcal{C}) \Longleftrightarrow(S \cap \mathcal{C}) \subseteq(T \cap \mathcal{C}) \\
\neg(S \cap \mathcal{C}):=<S \cap \mathcal{C}>^{\perp} \cap \mathcal{C}
\end{gathered}
$$

With these operations, we have that

Proposition 4.1. $\mathcal{L}$ is an atomic and complete lattice. If $\operatorname{dim}(\mathcal{H})<\infty, \mathcal{L}$ is a modular lattice. 
$\mathcal{L}$ is not an orthocomplemented lattice, but it is easy to show that non-contradiction holds

$$
L \wedge \neg L=\mathbf{0}
$$

and also contraposition

$$
L_{1} \leq L_{2} \Longrightarrow \neg L_{2} \leq L_{1}
$$

The following proposition links atoms and states:

Proposition 4.2. There is a one to one correspondence between the states of the system and the atoms of $\mathcal{L}$.

It is well known [21] that there is a lattice isomorphism between the complemented and complete lattice of faces of the convex set $\mathcal{C}$ and $\mathcal{L}_{v \mathcal{N}}$. Due to the following proposition

Proposition 4.3. Every face of $\mathcal{C}$ is an element of $\mathcal{L}$.

we conclude that

Corollary 4.4. The complete lattice of faces of the convex set $\mathcal{C}$ is a subposet of $\mathcal{L}$.

The previous Corollary shows that $\mathcal{L}$ and $\mathcal{L}_{v \mathcal{N}}$ are connected. What is the relationship between their operations? If $F_{1}$ and $F_{2}$ are faces we have:

$(\wedge) F_{1}, F_{2} \in \mathcal{L}_{v \mathcal{N}}$, then $F_{1} \wedge F_{2}$ in $\mathcal{L}_{v \mathcal{N}}$ is the same as in $\mathcal{L}$. So the inclusion $\mathcal{L}_{v \mathcal{N}} \subseteq \mathcal{L}$ preserves the $\wedge$-operation.

( $\vee F_{1} \vee_{\mathcal{L}} F_{2} \leq F_{1} \vee_{\mathcal{L}_{v \mathcal{N}}} F_{2}$ and $F_{1} \leq F_{2} \Rightarrow F_{1} \vee_{\mathcal{L}} F_{2}=F_{1} \vee_{\mathcal{L}_{v \mathcal{N}}} F_{2}=F_{2}$

$(\neg) \neg \mathcal{L} F \leq \neg \mathcal{L}_{v \mathcal{N}} F$

Given two systems with Hilbert spaces $\mathcal{H}_{1}$ and $\mathcal{H}_{2}$, we can construct the lattices $\mathcal{L}_{1}$ and $\mathcal{L}_{2}$. We can also construct $\mathcal{L}$, the lattice associated to the product space $\mathcal{H}_{1} \otimes \mathcal{H}_{2}$. We define:

$$
\Psi: \mathcal{L}_{1} \times \mathcal{L}_{2} \longrightarrow \mathcal{L} \quad \mid\left(S_{1} \cap \mathcal{C}_{1}, S_{2} \cap \mathcal{C}_{2}\right) \longrightarrow S \cap \mathcal{C}
$$

where $S=\left(<S_{1} \cap \mathcal{C}_{1}>\otimes<S_{2} \cap \mathcal{C}_{2}>\right)$. In terms of good representatives, $\Psi\left(\left[S_{1}\right],\left[S_{2}\right]\right)=$ $\left[S_{1} \otimes S_{2}\right]$. An equivalent way to define it (in the finite dimensional case) is by saying that $\Psi$ is the induced morphism in the quotient lattices of the tensor map

$$
G\left(\mathcal{A}_{1}\right) \times G\left(\mathcal{A}_{2}\right) \rightarrow G\left(\mathcal{A}_{1} \otimes_{\mathbb{R}} \mathcal{A}_{2}\right) \cong G(\mathcal{A})
$$

We can prove the following:

Proposition 4.5. Fixing $[U] \in \mathcal{L}_{2}$ then $\mathcal{L}_{1}$ is isomorphic (as complete lattice) to $\mathcal{L}_{1} \times[U] \subseteq \mathcal{L}$. The same is true for $\mathcal{L}_{2}$ and an arbitrary element of $\mathcal{L}_{1}$.

Given $L_{1} \in \mathcal{L}_{1}$ and $L_{2} \in \mathcal{L}_{2}$, we can define the following convex tensor product:

Definition 4.6. $L_{1} \widetilde{\otimes} L_{2}:=\left\{\sum \lambda_{i j} \rho_{i}^{1} \otimes \rho_{j}^{2} \mid \rho_{i}^{1} \in L_{1}, \rho_{j}^{2} \in L_{2}, \sum \lambda_{i j}=1\right.$ and $\left.\lambda_{i j} \geq 0\right\}$ 


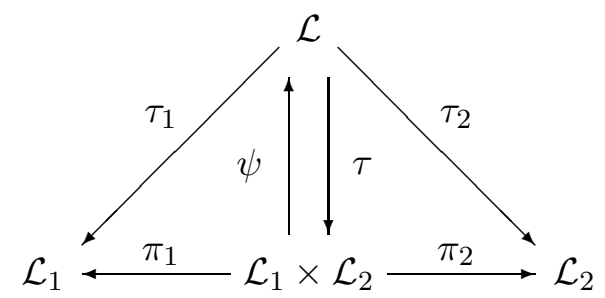

Figure 3: The different maps between $\mathcal{L}_{1}, \mathcal{L}_{2}, \mathcal{L}_{1} \times \mathcal{L}_{2}$, and $\mathcal{L} . \pi_{1}$ and $\pi_{2}$ are the canonical projections.

This product is formed by all possible convex combinations of tensor products of elements of $L_{1}$ and elements of $L_{2}$, and it is again a convex set. Let us compute $\mathcal{C}_{1} \widetilde{\otimes} \mathcal{C}_{2}$. Remember that $\mathcal{C}_{1}=\left[\mathcal{A}_{1}\right] \in \mathcal{L}_{1}$ and $\mathcal{C}_{2}=\left[\mathcal{A}_{2}\right] \in \mathcal{L}_{2}$ :

$$
\mathcal{C}_{1} \widetilde{\otimes} \mathcal{C}_{2}=\left\{\sum \lambda_{i j} \rho_{i}^{1} \otimes \rho_{j}^{2} \mid \rho_{i}^{1} \in \mathcal{C}_{1}, \rho_{j}^{2} \in \mathcal{C}_{2}, \quad \sum \lambda_{i j}=1 \text { and } \lambda_{i j} \geq 0\right\}
$$

So, if $\mathcal{S}(\mathcal{H})$ is the set of all separable states, we have by definition:

$$
\mathcal{S}(\mathcal{H})=\mathcal{C}_{1} \widetilde{\otimes} \mathcal{C}_{2}
$$

If the whole system is in a state $\rho$, using partial traces we can define states for the subsystems $\rho_{1}=\operatorname{tr}_{2}(\rho)$ and a similar definition for $\rho_{2}$. Then, we can consider the maps:

$$
\operatorname{tr}_{i}: \mathcal{C} \longrightarrow \mathcal{C}_{j} \quad \mid \rho \longrightarrow \operatorname{tr}_{i}(\rho)
$$

from which we can construct the induced projections:

$$
\tau_{i}: \mathcal{L} \longrightarrow \mathcal{L}_{i} \quad \mid \quad S \cap \mathcal{C} \longrightarrow \operatorname{tr}_{i}(<S \cap \mathcal{C}>) \cap \mathcal{C}_{i}
$$

In terms of good representatives $\tau_{i}([S])=\left[\operatorname{tr}_{i}(S)\right]$. Then we can define the product map

$$
\tau: \mathcal{L} \longrightarrow \mathcal{L}_{1} \times \mathcal{L}_{2} \quad \mid L \longrightarrow\left(\tau_{1}(L), \tau_{2}(L)\right)
$$

The maps defined in this section are shown in Figure 4 .

\section{The Lattice of Convex Subsets}

The elements of $\mathcal{L}$ are formed by intersections between closed subspaces and $\mathcal{C}$. Given that closed subspaces are closed sets and so is $\mathcal{C}$, they are also convex subsets of $\mathcal{C}$. We could go on further and consider all convex subsets of $\mathcal{C}$. On the other hand (because of linearity), partial trace operators preserve convexity and so they will map propositions of the system into propositions of the subsystem, as desired.

Another motivation for a further extension comes from the following analogy. If the propositions of classical mechanics are the subsets of $\Gamma$ (classical phase space), why cannot we consider the convex subsets of the convex set of states? It seems, after all, that convexity is an important feature of quantum mechanics [15], [16], and [17]. And as will be seen below, this "convexification" of the lattice, allows for an algebraic characterization of entanglement.

Let us begin by considering the set of all convex subsets of $\mathcal{C}$ :

Definition 5.1. $\mathcal{L}_{\mathcal{C}}:=\{C \subseteq \mathcal{C} \mid C$ is a convex subset of $\mathcal{C}\}$

In order to give $\mathcal{L}_{\mathcal{C}}$ a lattice structure, we introduce the following operations (where $\operatorname{conv}(A)$ stands for convex hull of a given set $A$ ):

Definition 5.2. For all $C, C_{1}, C_{2} \in \mathcal{L}_{\mathcal{C}}$ 


$$
\begin{aligned}
& \wedge C_{1} \wedge C_{2}:=C_{1} \cap C_{2} \\
& \vee C_{1} \vee C_{2}:=\operatorname{conv}\left(C_{1}, C_{2}\right) . \text { It is again a convex set, and it is included in } \mathcal{C} \text { (using convexity). } \\
& \neg \neg C:=C^{\perp} \cap \mathcal{C} \\
& \longrightarrow C_{1} \longrightarrow C_{2}:=C_{1} \subseteq C_{2}
\end{aligned}
$$

With the operations of definition $\left[5.2\right.$, it is apparent that $\left(\mathcal{L}_{\mathcal{C}} ; \longrightarrow\right)$ is a poset. If we set $\emptyset=\mathbf{0}$ and $\mathcal{C}=\mathbf{1}$, then, $\left(\mathcal{L}_{\mathcal{C}} ; \longrightarrow ; \mathbf{0} ; \emptyset=\mathbf{0}\right)$ will be a bounded poset.

Proposition 5.3. $\left(\mathcal{L}_{\mathcal{C}} ; \longrightarrow ; \wedge ; \vee\right)$ satisfies

(a) $C_{1} \wedge C_{1}=C_{1}$

(b) $C_{1} \wedge C_{2}=C_{2} \wedge C_{1}$

(c) $C_{1} \vee C_{2}=C_{2} \vee C_{1}$

(d) $\neg C:=C^{\perp} \cap \mathcal{C}$

(d) $C_{1} \wedge\left(C_{2} \wedge C_{3}\right)=\left(C_{1} \wedge C_{2}\right) \wedge C_{3}$

(e) $C_{1} \vee\left(C_{2} \vee C_{3}\right)=\left(C_{1} \vee C_{2}\right) \vee C_{3}$

(f) $C_{1} \wedge\left(C_{1} \vee C_{2}\right)=C_{1}$

(g) $C_{1} \vee\left(C_{1} \wedge C_{2}\right)=C_{1}$

Proof. $C_{1} \wedge C_{1}=C_{1} \cap C_{1}=C_{1}$, so we have $(a)$. (b), $(c)$ and $(d)$ are equally trivial. In order to prove $e$ we have that

$$
C_{1} \vee\left(C_{2} \vee C_{3}\right)=\operatorname{conv}\left(C_{1}, \operatorname{conv}\left(C_{2}, C_{3}\right)\right)
$$

Given that $\operatorname{conv}\left(C_{2}, C_{3}\right) \subseteq \operatorname{conv}\left(C_{1}, \operatorname{conv}\left(C_{2}, C_{3}\right)\right)$, then,

$$
C_{1}, C_{2}, C_{3} \subseteq \operatorname{conv}\left(C_{1}, \operatorname{conv}\left(C_{2}, C_{3}\right)\right)
$$

Using the above equation and convexity of $\operatorname{conv}\left(C_{1}, \operatorname{conv}\left(C_{2}, C_{3}\right)\right)$, we have that

$$
\operatorname{conv}\left(C_{1}, C_{2}\right) \subseteq \operatorname{conv}\left(C_{1}, \operatorname{conv}\left(C_{2}, C_{3}\right)\right)
$$

and so, using convexity,

$$
\operatorname{conv}\left(\operatorname{conv}\left(C_{1}, C_{2}\right), C_{3}\right) \subseteq \operatorname{conv}\left(C_{1}, \operatorname{conv}\left(C_{2}, C_{3}\right)\right)
$$

A similar argument implies the converse inclusion, and so we conclude that

$$
\left(C_{1} \vee C_{2}\right) \vee C_{3}=\operatorname{conv}\left(\operatorname{conv}\left(C_{1}, C_{2}\right), C_{3}\right)=\operatorname{conv}\left(C_{1}, \operatorname{conv}\left(C_{2}, C_{3}\right)\right)=C_{1} \vee\left(C_{2} \vee C_{3}\right)
$$

In order to prove $(f)$, we have $C_{1} \wedge\left(C_{1} \vee C_{2}\right)=C_{1} \cap \operatorname{conv}\left(C_{1}, C_{2}\right)$. As $C_{1} \cap \operatorname{conv}\left(C_{1}, C_{2}\right) \subseteq C_{1}$ and $C_{1} \subseteq \operatorname{conv}\left(C_{1}, C_{2}\right)$, we have $C_{1}=C_{1} \cap \operatorname{conv}\left(C_{1}, C_{2}\right)$, and so $(f)$ is true. Let us finally check (g). $C_{1} \vee\left(C_{1} \wedge C_{2}\right)=\operatorname{conv}\left(C_{1}, C_{1} \cap C_{2}\right)$. This implies that $C_{1}, C_{1} \cap C_{2} \subseteq \operatorname{conv}\left(C_{1}, C_{1} \cap C_{2}\right)$. As $C_{1}$ is convex, we have $\operatorname{conv}\left(C_{1}, C_{1} \cap C_{2}\right) \subseteq C_{1}$, and so we have $(g)$. 
Regarding the " $\neg$ " operation, if $C_{1} \subseteq C_{2}$, then $C_{2}^{\perp} \subseteq C_{1}^{\perp}$. So $C_{2}^{\perp} \cap \mathcal{C} \subseteq C_{1}^{\perp} \cap \mathcal{C}$, and hence

$$
C_{1} \longrightarrow C_{2} \Longrightarrow \neg C_{2} \longrightarrow \neg C_{1}
$$

Given that $C \cap\left(C^{\perp} \cap \mathcal{C}\right)=\emptyset$, we also have:

$$
C \wedge(\neg C)=\mathbf{0}
$$

and so, contraposition and non contradiction hold. But if we take the proposition $C=\left\{\frac{1}{N} 1\right\}$, then an easy calculation yields $\neg C=\mathbf{0}$. And then, $\neg(\neg C)=\mathbf{1}$, and thus $\neg(\neg C) \neq C$ in general. Double negation does not hold, thus, $\mathcal{L}_{\mathcal{C}}$ is not an ortholattice.

$\mathcal{L}_{\mathcal{C}}$ is a lattice which includes all convex subsets of the quantum space of states. It includes $\mathcal{L}$, and so, all quantum states (including all improper mixtures) as propositions. It is also in strong analogy with classical physics, where the lattice of propositions is formed by all measurable subsets of phase space (the space of states).

\subsection{The Relationship Between $\mathcal{L}_{v \mathcal{N}}, \mathcal{L}$ and $\mathcal{L}_{\mathcal{C}}$}

Proposition 5.4. $\mathcal{L}_{v \mathcal{N}} \subseteq \mathcal{L} \subseteq \mathcal{L}_{\mathcal{C}}$ as posets.

Proof. We have already seen that $\mathcal{L}_{v \mathcal{N}} \subseteq \mathcal{L}$ as sets. Moreover it is easy to see that if $F_{1} \leq F_{2}$ in $\mathcal{L}_{v \mathcal{N}}$ then $F_{1} \leq F_{2}$ in $\mathcal{L}$. This is so because both orders are set theoretical inclusions. Similarly, if $L_{1}, L_{2} \in \mathcal{L}$, because intersection of convex sets yields a convex set (and closed subspaces are convex sets also), $L_{1}, L_{2} \in \mathcal{L}_{\mathcal{C}}$, then we obtain set theoretical inclusion. And, again, because of both orders are set theoretical inclusions, we obtain that they are included as posets.

Regarding the $\vee$ operation, let us compare $\vee_{\mathcal{L}_{v \mathcal{N}}}, \vee_{\mathcal{L}}$ and $\vee_{\mathcal{L}_{C}}$. If $L_{1}, L_{2} \in \mathcal{L}$, then they are convex sets and so, $L_{1}, L_{2} \in \mathcal{L}_{\mathcal{C}}$. Then we can compute

$$
L_{1} \vee_{\mathcal{L}_{C}} L_{2}=\operatorname{conv}\left(L_{1}, L_{2}\right)
$$

On the other hand (if $S_{1}$ and $S_{2}$ are good representatives for $L_{1}$ and $L_{2}$ ), then:

$$
L_{1} \vee_{\mathcal{L}} L_{2}=\left(<S_{1} \cap \mathcal{C}>+<S_{2} \cap \mathcal{C}>\right) \cap \mathcal{C}
$$

The direct sum of the subspaces $\left\langle S_{1} \cap \mathcal{C}>\right.$ and $\left\langle S_{2} \cap \mathcal{C}\right\rangle$ contains as a particular case all convex combinations of elements of $L_{1}$ and $L_{2}$. So we can conclude

$$
L_{1} \vee_{\mathcal{L}_{C}} L_{2} \leq L_{1} \vee_{\mathcal{L}} L_{2}
$$

As faces of $\mathcal{C}$ can be considered as elements of $\mathcal{L}_{C}$ because they are convex, if $F_{1}$ and $F_{2}$ are faces, we can also state

$$
F_{1} \vee_{\mathcal{L}_{C}} F_{2} \leq F_{1} \vee_{\mathcal{L}} F_{2} \leq F_{1} \vee_{\mathcal{L}_{v \mathcal{N}}} F_{2}
$$

Intersection of convex sets is the same as intersection of elements of $\mathcal{L}$ and so we have

$$
L_{1} \wedge_{\mathcal{L}_{C}} L_{2}=L_{1} \wedge_{\mathcal{L}} L_{2}
$$

and similarly

$$
F_{1} \wedge \mathcal{L}_{v \mathcal{N}} F_{2}=F_{1} \wedge_{\mathcal{L}_{C}} F_{2}=F_{1} \wedge_{\mathcal{L}} F_{2}
$$

What is the relationship between $\neg \mathcal{L}_{\mathcal{C}}$ and $\neg \mathcal{L}$ ? Suppose that $L_{1} \in \mathcal{L}$, then they are convex sets also, and so $L_{1} \in \mathcal{L}_{\mathcal{C}}$. Then we can compute $\neg \mathcal{L}_{\mathcal{C}} L_{1}$. We obtain: 


$$
\neg \mathcal{L}_{\mathcal{C}} L_{1}=L_{1}^{\perp} \cap \mathcal{C}
$$

On the other hand, if $L_{1}=S \cap \mathcal{C}$, with $S$ a good representative

$$
\neg \mathcal{L}^{L_{1}}=<S \cap \mathcal{C}>^{\perp} \cap \mathcal{C}
$$

As $L_{1} \subseteq<S \cap \mathcal{C}>$, then $\left\langle S \cap \mathcal{C}>^{\perp} \subseteq L_{1}^{\perp}\right.$, and so

$$
\neg \mathcal{L} L_{1} \leq \neg \mathcal{L}_{\mathcal{C}} L_{1}
$$

\subsection{Interactions in $Q M$ and $C M$ Compared}

The origin of the extension of $\mathcal{L}_{v \mathcal{N}}$ becomes clear if wee make a comparison between classical and quantum compound systems. For a single classical system its properties are faithfully represented by the subsets of its phase space. When another classical system is added and the compound system is considered, no enrichment of the state space of the former system is needed in order to describe its properties, even in the presence of interactions. No matter which the interactions may be, the cartesian product of phase spaces is sufficient for the description of the compound system.

The situation is quite different in quantum mechanics. This is so because, if we add a new quantum system to a previously isolated one, pure states are no longer faithful in order to describe subsystems. Interactions produce non trivial correlations, which are reflected in the presence of entangled states (and violation of Bell inequalities). Thus, we have to consider the information about the non trivial correlations that each subsystem has with other subsystems -for example, a system with the environment. The existence of this additional information implies that we must add new elements to the propositional structure of the system.

\section{The Relationship Between $\mathcal{L}_{\mathcal{C}}$ and The Tensor Product of Hilbert Spaces}

In this section we study the relationship between the lattice $\mathcal{L}_{\mathcal{C}}$ of a system $S$ composed of subsystems $S_{1}$ and $S_{2}$, and the lattices of its subsystems, $\mathcal{L}_{\mathcal{C} 1}$ and $\mathcal{L}_{\mathcal{C} 2}$ respectively. As in [14], we do this by making the physical interpretation of maps which can be defined between them.

\subsection{Separable States (Going Up)}

Let us define:

Definition 6.1. Given $C_{1} \subseteq \mathcal{C}_{1}$ and $C_{2} \subseteq \mathcal{C}_{2}$

$$
C_{1} \otimes C_{2}:=\left\{\rho_{1} \otimes \rho_{2} \mid \rho_{1} \in C_{1}, \rho_{2} \in C_{2}\right\}
$$

Then, we define the map:

\section{Definition 6.2.}

$$
\begin{gathered}
\Lambda: \mathcal{L}_{\mathcal{C} 1} \times \mathcal{L}_{\mathcal{C} 2} \longrightarrow \mathcal{L}_{\mathcal{C}} \\
\left(C_{1}, C_{2}\right) \longrightarrow \operatorname{conv}\left(C_{1} \otimes C_{2}\right)
\end{gathered}
$$

In the rest of this work will use the following proposition (see for example [24]): 
Proposition 6.3. Let $S$ be a subset of a linear space $\mathbb{L}$. Then $x \in \operatorname{conv}(S)$ iff $x$ is contained in a finite dimensional simplex $\Delta$ whose vertices belong to $S$.

From equation 4.0.30 and definition 4.6 it should be clear that $\Lambda\left(\mathcal{C}_{1}, \mathcal{C}_{2}\right)=\mathcal{S}(\mathcal{H})$. Definition 4.6 also implies that for all $C_{1} \subseteq \mathcal{C}_{1}$ and $C_{2} \subseteq \mathcal{C}_{2}$ :

$$
\Lambda\left(C_{1}, C_{2}\right)=C_{1} \widetilde{\otimes} C_{2}
$$

Proposition 6.4. Let $\rho=\rho_{1} \otimes \rho_{2}$, with $\rho_{1} \in \mathcal{C}_{1}$ and $\rho_{2} \in \mathcal{C}_{2}$. Then $\{\rho\}=\Lambda\left(\left\{\rho_{1}\right\},\left\{\rho_{2}\right\}\right)$ with $\left\{\rho_{1}\right\} \in \mathcal{L}_{C 1},\left\{\rho_{2}\right\} \in \mathcal{L}_{C 2}$ and $\{\rho\} \in \mathcal{C}$.

Proof. We already know that the atoms are elements of the lattices. Thus,

$$
\Lambda\left(\left\{\rho_{1}\right\},\left\{\rho_{2}\right\}\right)=\operatorname{conv}\left(\left\{\rho_{1} \otimes \rho_{2}\right\}\right)=\left\{\rho_{1} \otimes \rho_{2}\right\}=\{\rho\}
$$

Proposition 6.5. Let $\rho \in \mathcal{S}(\mathcal{H})$, the set of separable states. Then, there exist $C \in \mathcal{L}_{\mathcal{C}}, C_{1} \in \mathcal{L}_{\mathcal{C}_{1}}$ and $C_{2} \in \mathcal{L}_{C_{2}}$ such that $\rho \in C$ and $L=\Lambda\left(C_{1}, C_{2}\right)$.

Proof. If $\rho \in \mathcal{S}(\mathcal{H})$, then $\rho=\sum_{i j} \lambda_{i j} \rho_{i}^{1} \otimes \rho_{j}^{2}$, with $\sum_{i j} \lambda_{i j}=1$ and $\lambda_{i j} \geq 0$. Consider the convex sets:

$$
C_{1}=\operatorname{conv}\left(\left\{\rho_{1}^{1}, \rho_{2}^{1}, \cdots, \rho_{k}^{1}\right\}\right) \quad C_{2}=\operatorname{conv}\left(\left\{\rho_{1}^{2}, \rho_{2}^{2}, \cdots, \rho_{l}^{2}\right\}\right)
$$

Then we define:

$$
C:=\Lambda\left(C_{1}, C_{2}\right)=\operatorname{conv}\left(C_{1} \otimes C_{2}\right)
$$

Clearly, the set $\left\{\rho_{i}^{1} \otimes \rho_{j}^{2}\right\} \subseteq C_{1} \otimes C_{2}$, and then $\rho \in C$.

\subsection{Projections Onto $\mathcal{L}_{\mathcal{C}_{1}}$ and $\mathcal{L}_{\mathcal{C}_{2}}$ (Going Down)}

Let us now study the projections onto $\mathcal{L}_{\mathcal{C}_{1}}$ and $\mathcal{L}_{\mathcal{C}_{2}}$. From a physical point of view, it is of interest to study the partial trace operators. If the whole system is in a state $\rho$, using partial traces we can define states for the subsystems $\rho_{1}=\operatorname{tr}_{2}(\rho)$ and a similar definition for $\rho_{2}$. Then, we can consider the maps:

$$
\operatorname{tr}_{i}: \mathcal{C} \longrightarrow \mathcal{C}_{j} \quad \mid \quad \rho \longrightarrow \operatorname{tr}_{i}(\rho)
$$

from which we can construct the induced projections:

$$
\tau_{i}: \mathcal{L}_{\mathcal{C}} \longrightarrow \mathcal{L}_{\mathcal{C}_{i}} \quad \mid \quad C \longrightarrow \operatorname{tr}_{i}(C)
$$

Then we can define the product map

$$
\tau: \mathcal{L}_{\mathcal{C}} \longrightarrow \mathcal{L}_{\mathcal{C}_{1}} \times \mathcal{L}_{\mathcal{C}_{2}} \quad \mid \quad C \longrightarrow\left(\tau_{1}(C), \tau_{2}(C)\right)
$$

We use the same notation for $\tau$ and $\tau_{i}$ (though they are different functions) as in 14 and section 4, and this should not introduce any difficulty. We can prove the following about the image of $\tau_{i}$.

Proposition 6.6. The maps $\tau_{i}$ preserve the convex structure, i.e., they map convex sets into convex sets. 
Proof. Let $C \subseteq \mathcal{C}$ be a convex set. Let $C_{1}$ be the image of $C$ under $\tau_{2}$ (a similar argument holds for $\tau_{1}$ ). Let us show that $C_{1}$ is convex. Let $\rho_{1}$ and $\rho_{1}^{\prime}$ be elements of $C_{1}$. Consider $\sigma_{1}=\alpha \rho_{1}+(1-\alpha) \rho_{1}^{\prime}$, with $0 \leq \alpha \leq 1$. Then, there exists $\rho, \rho^{\prime} \in \mathcal{C}$ such that:

$$
\sigma_{1}=\alpha \operatorname{tr}_{2}(\rho)+(1-\alpha) \operatorname{tr}_{2}\left(\rho^{\prime}\right)=\operatorname{tr}_{2}\left(\alpha \rho+(1-\alpha) \rho^{\prime}\right)
$$

where we have used the linearity of trace. Because of convexity of $C, \sigma:=\alpha \rho+(1-\alpha) \rho^{\prime} \in C$, and so, $\sigma_{1}=\operatorname{tr}_{2}(\sigma) \in C_{1}$.

Proposition 6.7. The functions $\tau_{i}$ are surjective and preserve the $\vee$-operation. They are not injective.

Proof. Take the convex set $C_{1} \in \mathcal{L}_{\mathcal{C}_{1}}$. Choose an arbitrary element of $\mathcal{C}_{2}$, say $\rho_{2}$. Now consider the following element of $\mathcal{L}_{\mathcal{C}}$

$$
C=C_{1} \otimes \rho_{2}
$$

$C$ is convex, and so belongs to $\mathcal{L}_{\mathcal{C}}$, because if $\rho \otimes \rho_{2}, \sigma \otimes \rho_{2} \in C$, then any convex combination $\alpha \rho \otimes \rho_{2}+(1-\alpha) \sigma \otimes \rho_{2}=(\alpha \rho+(1-\alpha) \sigma) \otimes \rho_{2} \in C$ (where we have used convexity of $C_{1}$ ). It is clear that $\tau_{1}(C)=C_{1}$, because if $\rho_{1} \in C_{1}$, then $\operatorname{tr}\left(\rho_{1} \otimes \rho_{2}\right)=\rho_{1}$. So, $\tau_{1}$ is surjective. On the other hand, the arbitrariness of $\rho_{2}$ implies that it is not injective. An analogous argument follows for $\tau_{2}$.

Let us see that $\tau_{i}$ preserves the $\vee$-operation. Let $C$ and $C^{\prime}$ be convex subsets of $\mathcal{C}$. We must compute $\left.\operatorname{tr}_{2}\left(C \vee C^{\prime}\right)\right)=\operatorname{tr}_{2}\left(\operatorname{conv}\left(C, C^{\prime}\right)\right)$. We must show that this is the same as $\operatorname{conv}\left(\operatorname{tr}_{2}(C), \operatorname{tr}_{2}\left(C^{\prime}\right)\right)$. Take $x \in \operatorname{conv}\left(\operatorname{tr}_{2}(C), \operatorname{tr}_{2}\left(C^{\prime}\right)\right)$. Then $x=\alpha \operatorname{tr}_{2}(\rho)+(1-\alpha) \operatorname{tr}_{2}\left(\rho^{\prime}\right)$, with $\rho \in C, \rho^{\prime} \in C^{\prime}$ and $0 \leq \alpha \leq 1$. Using linearity of trace, $x=\operatorname{tr}_{2}\left(\alpha \rho+(1-\alpha) \rho^{\prime}\right)$. $\alpha \rho+(1-\alpha) \rho^{\prime} \in \operatorname{conv}\left(C, C^{\prime}\right)$, and so, $x \in \operatorname{tr}_{2}\left(\operatorname{conv}\left(C, C^{\prime}\right)\right)$. Hence we have

$$
\operatorname{conv}\left(\operatorname{tr}_{2}(C), \operatorname{tr}_{2}\left(C^{\prime}\right)\right) \subseteq \operatorname{tr}_{2}\left(\operatorname{conv}\left(C, C^{\prime}\right)\right)
$$

In other to prove the other inclusion, take $x \in \operatorname{tr}_{2}\left(\operatorname{conv}\left(C, C^{\prime}\right)\right)$. Then,

$$
x=\operatorname{tr}_{2}\left(\alpha \rho+(1-\alpha) \rho^{\prime}\right)=\alpha \operatorname{tr}_{2}(\rho)+(1-\alpha) \operatorname{tr}_{2}\left(\rho^{\prime}\right)
$$

with $\rho \in C_{1}$ and $\rho^{\prime} \in C^{\prime}$. On the other hand, $\operatorname{tr}_{2}(\rho) \in \operatorname{tr}_{2}(C)$ and $\operatorname{tr}_{2}\left(\rho^{\prime}\right) \in \operatorname{tr}_{2}\left(C^{\prime}\right)$. This proves that:

$$
\operatorname{tr}_{2}\left(\operatorname{conv}\left(C, C^{\prime}\right)\right) \subseteq \operatorname{conv}\left(\operatorname{tr}_{2}(C), \operatorname{tr}_{2}\left(C^{\prime}\right)\right)
$$

Let us now consider the $\wedge$-operation. If $x \in \tau_{i}\left(C \wedge C^{\prime}\right)=\tau_{i}\left(C \cap C^{\prime}\right)$ then $x=\tau_{i}(\rho)$ with $\rho \in C \cap C^{\prime}$. But if $\rho \in C$, then $x=\tau_{i}(\rho) \in \operatorname{tr}_{i}(C)$. As $\rho \in C^{\prime}$ also, a similar argument shows that $x=\tau_{i}(\rho) \in \operatorname{tr}_{i}\left(C^{\prime}\right)$. Then $x \in \tau_{i}(C) \cap \tau_{i}\left(C^{\prime}\right)$. And so:

$$
\tau_{i}\left(C \cap C^{\prime}\right) \subseteq \tau_{i}(C) \cap \tau_{i}\left(C^{\prime}\right)
$$

which is the same as:

$$
\tau_{i}\left(C \wedge C^{\prime}\right) \leq \tau_{i}(C) \wedge \tau_{i}\left(C^{\prime}\right)
$$

But these sets are not equal in general, as the following example shows. Take $\left\{\rho_{1} \otimes \rho_{2}\right\} \in \mathcal{L}$ and $\left\{\rho_{1} \otimes \rho_{2}^{\prime}\right\} \in \mathcal{L}$, with $\rho^{\prime} \neq \rho$. It is clear that $\left\{\rho_{1} \otimes \rho_{2}\right\} \wedge\left\{\rho_{1} \otimes \rho_{2}^{\prime}\right\}=\mathbf{0}$ and so, $\tau_{1}\left(\left\{\rho_{1} \otimes\right.\right.$ 


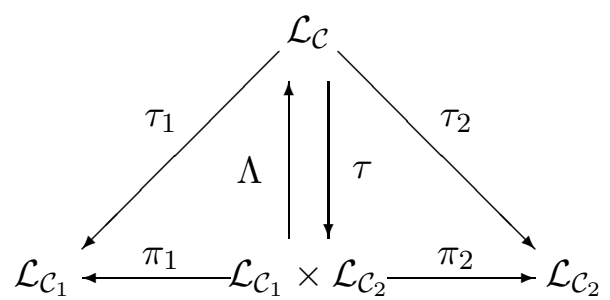

Figure 4: The different maps between $\mathcal{L}_{\mathcal{C}_{1}}, \mathcal{L}_{\mathcal{C}_{2}}, \mathcal{L}_{\mathcal{C}_{1}} \times \mathcal{L}_{\mathcal{C}_{2}}$, and $\mathcal{L}_{\mathcal{C}}$

$\left.\left.\rho_{2}\right\} \wedge\left\{\rho_{1} \otimes \rho_{2}^{\prime}\right\}\right)=\mathbf{0}$. On the other hand, $\tau_{1}\left(\left\{\rho_{1} \otimes \rho_{2}\right\}\right)=\left\{\rho_{1}\right\}=\tau_{1}\left(\left\{\rho_{1} \otimes \rho_{2}^{\prime}\right\}\right)$, and so, $\tau_{1}\left(\left\{\rho_{1} \otimes \rho_{2}\right\}\right) \wedge \tau_{1}\left(\left\{\rho_{1} \otimes \rho_{2}^{\prime}\right\}\right)=\left\{\rho_{1}\right\}$. A similar fact holds for the $\neg$-operation.

The last result is in strong analogy with what happens in $\mathcal{L}$, where lack of injectivity of the $\tau_{i}$ may be physically interpreted in the fact that the whole system has much more information than that of its parts. It is again useful to make a comparison with the classical case in order to illustrate what is happening. The same as in classical mechanics, we have atoms in $\mathcal{L}$ which are tensor products of atoms of $\mathcal{L}_{1}$ and $\mathcal{L}_{2}$. But in contrast to classical mechanics, entangled states originate atoms of $\mathcal{L}$ which cannot be expressed in such a way, and thus, the fiber of the projection $\tau_{i}$ is much bigger than that of its classical counterpart.

It is again an important result that the projection function $\tau$ cannot be defined properly within the frame of the traditional approaches of $Q L$ because there is no place for improper mixtures in those formalisms. But in the formalism presented here they are included as elements of the lattices, and so we can define the projections from the lattice of the whole system to the lattices of the subsystems. This enables a more natural approach when compound systems are considered from a quantum logical point of view.

\subsection{An Algebraic Characterization for Entanglement}

We shown that it is possible to extend $\mathcal{L}_{v \mathcal{N}}$ in order to deal with statistical mixtures and that $\mathcal{L}$ and $\mathcal{L}_{\mathcal{C}}$ are possible extensions. It would be interesting to search for a characterization of entanglement within this framework. Let us see first what happens with the functions $\Lambda \circ \tau$ and $\tau \circ \Lambda$. We have:

Proposition 6.8. $\tau \circ \Lambda=I d$.

Proof.

$$
\begin{aligned}
& \tau_{1}\left(\Lambda\left(C_{1}, C_{2}\right)\right)=\tau_{1}\left(\operatorname{conv}\left(C_{1} \otimes C_{2}\right)\right)=\operatorname{tr}_{2}\left(\operatorname{conv}\left(C_{1} \otimes C_{2}\right)\right)=C_{1} \\
& \tau_{2}\left(\Lambda\left(C_{1}, C_{2}\right)\right)=\tau_{2}\left(\operatorname{conv}\left(C_{1} \otimes C_{2}\right)\right)=\operatorname{tr}_{1}\left(\operatorname{conv}\left(C_{1} \otimes C_{2}\right)\right)=C_{2}
\end{aligned}
$$

Then $\tau\left(\Lambda\left(C_{1}, C_{2}\right)\right)=\left(C_{1}, C_{2}\right)$.

Again, as in 14, if we take into account physical considerations, $\Lambda \circ \tau$ is not the identity function. This is because when we take partial traces, we face the risk of losing information which will not be recovered when we make products of states. So we obtain the same slogan as before [14: "going down and then going up is not the same as going up and then going down". We show these maps in Figure 4. How is this related to entanglement? If we restrict $\Lambda \circ \tau$ to the set of product states, then it reduces to the identity function, for if $\rho=\rho_{1} \otimes \rho_{2}$, then:

$$
\Lambda \circ \tau(\{\rho\})=\{\rho\}
$$

On the other hand, it should be clear that if $\rho$ is an entangled state

$$
\Lambda \circ \tau(\{\rho\}) \neq\{\rho\}
$$

because $\Lambda \circ \tau(\{\rho\})=\left\{\operatorname{Tr}_{2}(\rho) \otimes \operatorname{Tr}_{1}(\rho)\right\} \neq\{\rho\}$ for any entangled state. This property points in the direction of an arrow characterization of entanglement. There are mixed states which are 
not product states, and so, entangled states are not the only ones who satisfy equation 6.3.2. What is the condition satisfied for a general mixed state? The following proposition summarizes all of this.

Proposition 6.9. If $\rho$ is a separable state, then there exists a convex set $S_{\rho} \subseteq \mathcal{S}(\mathcal{H})$ such that $\rho \in S_{\rho}$ and $\Lambda \circ \tau\left(S_{\rho}\right)=S_{\rho}$. More generally, for a convex set $C \subseteq \mathcal{S}(\mathcal{H})$, then there exists a convex set $S_{C} \subseteq \mathcal{S}(\mathcal{H})$ such that $\Lambda \circ \tau\left(S_{C}\right)=S_{C}$. For a product state, we can choose $S_{\rho}=\{\rho\}$. Any proposition $C \in \mathcal{L}_{\mathcal{C}}$ which has at lest one non-separable state, satisfies that there is no convex set $S$ such that $C \subseteq S$ and $\Lambda \circ \tau(S)=S$.

Proof. We have already seen above that if $\rho$ is a product state, then $\Lambda \circ \tau(\{\rho\})=\{\rho\}$, and so $S_{\rho}=\{\rho\}$. If $\rho$ is a general separable state, then there exists $\rho_{1 k} \in \mathcal{C}_{1}, \rho_{2 k} \in \mathcal{C}_{1}$ and $\alpha_{k} \geq 0, \sum_{k=1}^{N} \alpha_{k}=1$ such that $\rho=\sum_{k=1}^{N} \alpha_{k} \rho_{1 k} \otimes \rho_{2 k}$. Now consider the convex set (a simplex)

$$
M=\left\{\sigma \in \mathcal{C} \mid \sigma=\sum_{i, j=1}^{N} \lambda_{i, j} \rho_{1 i} \otimes \rho_{2 j}, \lambda_{i, j} \geq 0, \sum_{i, j=1}^{N} \lambda_{i, j}=1\right\}
$$

It is formed by all convex combinations of products of the elements which appear in the decomposition of $\rho$. It should be clear that $\rho \in M$. If we apply $\left(\operatorname{tr}_{1}(), \operatorname{tr}_{2}()\right)$ to $\sigma \in M$, we get

$$
\left(\operatorname{tr}_{1}(\sigma), \operatorname{tr}_{2}(\sigma)\right)=\left(\sum_{i=1}^{N}\left(\sum_{j=1}^{N} \lambda_{i, j}\right) \rho_{1 i}, \sum_{j=1}^{N}\left(\sum_{i=1}^{N} \lambda_{i, j}\right) \rho_{2 j}\right)=\left(\sum_{i=1}^{N} \mu_{i} \rho_{1 i}, \sum_{j=1}^{N} \nu_{j} \rho_{2 j}\right)
$$

with $\mu_{i}=\sum_{j=1}^{N} \lambda_{i, j}$ and $\nu_{i}=\sum_{i=1}^{N} \lambda_{i, j}$. Note that $\sum_{j=1}^{N} \mu_{j}=\sum_{j=1}^{N} \nu_{j}=1$. If we now apply $\Lambda$ :

$$
\Lambda\left(\left(\sum_{i=1}^{N} \mu_{i} \rho_{1 i}, \sum_{j=1}^{N} \nu_{j} \rho_{2 j}\right)\right)=\sum_{i, j=1}^{N} \mu_{i} \nu_{j} \rho_{1 i} \otimes \rho_{2 j}
$$

which is an element of $M$, and so, we conclude that $\Lambda \circ \tau(M) \subseteq M$. On the other hand, if $\sigma \in M$, then $\sigma=\sum_{i, j=1}^{N} \lambda_{i, j} \rho_{1 i} \otimes \rho_{2 j}$ (convex combination). It is important to note that $\Lambda \circ \tau(M)$ is a convex set, because trace operators preserve convexity, and $\Lambda$ is a convex hull. On the other hand $\Lambda \circ \tau\left(\left\{\rho_{1 i} \otimes \rho_{2 j}\right\}\right)=\left\{\rho_{1 i} \otimes \rho_{2 j}\right\}$. And so, by convexity of $\Lambda \circ \tau(M), \sigma \in \Lambda \circ \tau(M)$. Finally, $\Lambda \circ \tau(M)=M$ (and $\rho \in M)$. Then $M$ is the desired $S_{\rho} \subseteq \mathcal{S}(\mathcal{H})$.

If $C \subseteq \mathcal{S}(\mathcal{H})$, then all $\rho \in C$ are separable. $\mathcal{S}(\mathcal{H})$ is by definition, a convex set. Let us see that it is invariant under $\Lambda \circ \tau$. First of all, we know that $\mathcal{S}(\mathcal{H})$ is formed by all possible convex combinations of the from $\rho_{1} \otimes \rho_{2}$, with $\rho_{1} \in \mathcal{C}_{1}$ and $\rho_{2} \in \mathcal{C}_{2}$. But for each one of these tensor products, $\Lambda \circ \tau\left(\left\{\rho_{1} \otimes \rho_{2}\right\}\right)=\left\{\rho_{1} \otimes \rho_{2}\right\}$, and so belongs to $\Lambda \circ \tau(\mathcal{S}(\mathcal{H}))$. This is a convex set, thus all convex combinations of them belong to it. So we can conclude that

$$
\Lambda \circ \tau(\mathcal{S}(\mathcal{H}))=\mathcal{S}(\mathcal{H})
$$

Now, consider $C \in \mathcal{L}_{\mathcal{C}}$ such that there exists $\rho \in C$, being $\rho$ nonseparable. $\Lambda \circ \tau(S) \subseteq \mathcal{S}(\mathcal{H})$ for all $S \in \mathcal{L}_{\mathcal{C}}$. Then, it could never happen that there exists $S \in \mathcal{L}_{\mathcal{C}}$ such that $C \subseteq S$ and $\Lambda \circ \tau(S)=S$. 
From the last proposition, we conclude that there is a property which the convex subsets of separable states satisfy, and convex subsets which include non-separable states do not. This motivates the following definition.

Definition 6.10. If $C \in \mathcal{L}_{\mathcal{C}}$, we will say that it is a separable proposition if there exists $S_{C} \in \mathcal{L}_{\mathcal{C}}$ such that $\Lambda \circ \tau\left(S_{C}\right)=S_{C}$ and $C \subseteq S$. Otherwise, we will say that it is a non-separable or entangled proposition.

\subsection{The Inverse $\tau$-map}

In section 6.2 we defined the function $\tau=\left(\tau_{1}, \tau_{2}\right)$. Now we show that using the inverse map $\tau^{-1}=\left(\tau_{1}^{-1}, \tau_{2}^{-1}\right)$ we obtain lattice morphisms. It is easy to show that $\tau_{i}^{-1}$ maps any proposition from $\mathcal{C}_{i}$ into a proposition of $\mathcal{C}$. This is because the pre-image of a convex set under these functions is again a convex set. If $C_{1}$ is a proposition of $\mathcal{C}$ and if $\tau_{1}(\rho), \tau_{1}\left(\rho^{\prime}\right) \in C_{1}$, it is clear that any convex combination of $\rho$ and $\rho^{\prime}$ will belong to $\tau_{1}^{-1}\left(C_{1}\right)$, because the partial trace is linear and $C_{1}$ is convex.

Proposition 6.11. For all $X \in \mathcal{L}_{C} X \subseteq \tau_{1}^{-1}\left(\tau_{1}(X)\right)$ and for all $Y \in \mathcal{L}_{C_{1}}, \tau_{1}\left(\tau_{1}^{-1}(Y)\right)$. For all $C \subseteq \mathcal{C}$ we have $C \subseteq \tau_{1}^{-1}\left(C_{1}\right) \wedge \tau_{2}^{-1}\left(C_{2}\right)$

Proof. Let $X \in \mathcal{L}_{C}$. Then, if $x \in X$ it follows that $\tau_{1}(x) \in \tau_{1}(X)$ and so, $X \subseteq \tau_{1}^{-1}\left(\tau_{1}(X)\right)$. If $Y \in \mathcal{L}_{C_{1}}$ and $z \in \tau_{1}\left(\tau_{1}^{-1}(Y)\right)$. Then by definition of $\tau_{1}^{-1}(Y)$, it follows that $z \in Y$.

Let $C \in \mathcal{L}_{\mathcal{C}}$. Now $\tau_{1}(C)=C_{1} \in \mathcal{L}_{\mathcal{C}_{1}}$ and $\tau_{2}(C)=C_{2} \in \mathcal{L}_{\mathcal{C}_{2}}$. Then, it is apparent that $C \subseteq \tau_{1}^{-1}\left(C_{1}\right)$ and $C \subseteq \tau_{2}^{-1}\left(C_{2}\right)$. And so $C \subseteq \tau_{1}^{-1}\left(C_{1}\right) \wedge \tau_{2}^{-1}\left(C_{2}\right)$.

Proposition 6.12. For all $a, b \in \mathcal{L}_{\mathcal{C}_{1}} \tau_{1}^{-1}(a \wedge b)=\tau_{1}^{-1}(a) \wedge \tau_{1}^{-1}(b), \tau_{1}^{-1}(a \vee b)=\tau_{1}^{-1}(a) \vee \tau_{1}^{-1}(b)$. Furthermore, $\tau_{1}^{-1}$ is an injective function and if $a, b \in \mathcal{L}_{\mathcal{C}_{1}}$ and $a \subseteq b$, then $\tau_{1}^{-1}(a) \subseteq \tau_{1}^{-1}(b)$. If $\rho \neq \rho^{\prime}$ then $\tau_{1}^{-1}(\rho) \wedge \tau_{1}^{-1}\left(\rho^{\prime}\right)=\mathbf{0}$.

Proof. Consider the sets $\tau_{1}^{-1}(a \wedge b)$ and $\tau_{1}^{-1}(a) \wedge \tau_{1}^{-1}(b)$. Then, $x \in \tau_{1}^{-1}(a)$ and $x \in \tau_{1}^{-1}(b)$. If $x \in \tau_{1}^{-1}(a \wedge b)$, then $\tau_{1}(x) \in a \wedge b \subseteq a$, and we obtain also $\tau_{1}(x) \in a \wedge b \subseteq b$. This means that $x \in \tau_{1}^{-1}(a)$ and $x \in \tau_{1}^{-1}(b)$. So we have $\tau_{1}^{-1}(a \wedge b) \subseteq \tau_{1}^{-1}(a) \wedge \tau_{1}^{-1}(b)$. On the other hand, if $x \in \tau_{1}^{-1}(a) \wedge \tau_{1}^{-1}(b)$, then $x \in \tau_{1}^{-1}(a)$ and $x \in \tau_{1}^{-1}(b)$. This means that $\tau_{1}(x) \in a$ and $\tau_{1}(x) \in b$, and so, $\tau_{1}(x) \in a \wedge b$. This means that $x \in \tau_{1}^{-1}(a \wedge b)$. This concludes the proof that $\tau_{1}^{-1}(a \wedge b)=\tau_{1}^{-1}(a) \wedge \tau_{1}^{-1}(b)$.

If $x \in \tau_{1}^{-1}(a) \vee \tau_{1}^{-1}(b)$ then $x=\alpha \rho+\beta \rho^{\prime}$, with $\tau_{1}(\rho) \in a$ and $\tau_{1}\left(\rho^{\prime}\right) \in b$. So $\tau_{1}(x)=$ $\alpha \tau_{1}(\rho)+\beta \tau_{1}\left(\rho^{\prime}\right) \in a \vee b$. This means that $x \in \tau_{1}^{-1}(a \vee b)$, and we have $\tau_{1}^{-1}(a \vee b) \supseteq \tau_{1}^{-1}(a) \vee \tau_{1}^{-1}(b)$. Now, let $x \in \tau_{1}^{-1}(a \vee b)$. Then, $\tau_{1}(x) \in a \vee b$. This means that $\tau_{1}(x)=\alpha \rho+\beta \rho^{\prime}$ (convex combination), with $\rho \in a$ and $\rho^{\prime} \in b$. There exist $\sigma \in \tau_{1}^{-1}(a)$ and $\sigma^{\prime} \in \tau_{1}^{-1}(b)$ such that $\tau_{1}(\sigma)=\rho$ and $\tau_{1}\left(\sigma^{\prime}\right)=\rho^{\prime}$. Then $\tau_{1}(x)=\alpha \tau_{1}(\sigma)+\beta \tau_{1}\left(\sigma^{\prime}\right) . \tau_{1}()$ is a linear function so, the last equality implies $\tau_{1}\left(x-\left(\alpha \sigma+\beta \sigma^{\prime}\right)\right)=0$. Then, there exists $\varsigma \in \operatorname{Ker}\left(\tau_{1}()\right)$ such that $x=\alpha \sigma+\beta \sigma^{\prime}+\varsigma$. If $\beta=0$, then $\alpha=1$ (convex combination), and then, $x=\sigma \in \tau_{1}^{-1}(a)$, and in that case $x \in \tau_{1}^{-1}(a) \vee \tau_{1}^{-1}(b)$. If $\beta \neq 0$, we can put $x=\alpha \sigma+\beta\left(\sigma^{\prime}+\frac{1}{\beta} \varsigma\right) . \tau_{1}\left(\left(\sigma^{\prime}+\frac{1}{\beta} \varsigma\right)\right)=\tau_{1}\left(\sigma^{\prime}\right)+0 \in b$, and so $\sigma^{\prime}+\frac{1}{\beta} \varsigma \in \tau_{1}^{-1}(b)$. This proves that $x \in \tau_{1}^{-1}(a) \vee \tau_{1}^{-1}(b)$, and thus $\tau_{1}^{-1}(a \vee b) \subseteq \tau_{1}^{-1}(a) \vee \tau_{1}^{-1}(b)$ 
Let $a$ and $b$ be two propositions such that $a \neq b$. Suppose that $\tau_{1}^{-1}(a)=\tau_{1}^{-1}(b)$. If $a \neq b$, there exists $\rho_{a} \in a$ such that $\rho_{a} \notin b$. It is clear that $\tau_{1}^{-1}\left(\rho_{a}\right) \subseteq \tau_{1}^{-1}(a)=\tau_{1}^{-1}(b)$ and then, there exists $\rho \in \tau_{1}^{-1}(b)$ such that $\tau_{1}(\rho)=\rho_{a}$. But by definition of $\tau_{1}^{-1}(b)$, we would have that $\rho_{a} \in b$, a contradiction. Thus, we have $\tau_{1}^{-1}(a) \neq \tau_{1}^{-1}(b)$.

If $a \subseteq b$, suppose that $x \in \tau_{1}^{-1}(a)$. Then $\tau_{1}(x) \in b$, and so $x \in \tau_{1}^{-1}(b)$ also. If $x \in \tau_{1}^{-1}(\rho)$, $x \in \tau_{1}^{-1}\left(\rho^{\prime}\right)$ and $\rho \neq \rho^{\prime}$, then $\rho=\tau_{1}(x)=\rho^{\prime}$, a contradiction.

\section{The Difference with Other Approaches and Conclusions}

The problem of compound quantum systems has been widely studied from different approaches. An important difference of our approach is that it treats improper mixtures in a different way. In this work, we made the following reasoning line. In $C M$ the fundamental description is given by subsets (propositions) of the phase space. Statistical mixtures are not fundamental; they appear as a limitation in the capability of knowledge of the observer. It is for that reason that they are expressed as proper mixtures, and so in the orthodox logical approach they appear in different levels: the propositions lie in the lattice $\mathcal{L}_{\mathcal{C M}}$, while the mixtures are measures over this lattice. Pure states are in one to one correspondence with the atoms of the lattice, and so they are also included as elements of the lattice, but mixtures are in a different level.

The situation turns radically different in $Q M$ if we accept that improper mixtures do not admit an ignorance interpretation. If $S_{1}$ is in a state represented by an improper mixture $\rho$, there is no more physical information available for the observer. $\rho$ represents the actual state for $S_{1}$ and we cannot get more information, not because of our experimental limitations, but because of that information does not exist. Thus, they should be represented at the same level as that of pure states, because they are maximal pieces of information.

On the other hand, influenced by historical reasons, the orthodox $Q L$ approach, still retains the analogy with $C M$ and considers improper mixtures in a different level than that of the propositions. Thus, the orthodox $Q L$ approach presents some difficulties when compound quantum systems are involved. We studied these problems in section 3 and we gave a list of conditions on the structures that we are looking for in order to solve these difficulties.

The structures presented in this work and in [14], $\mathcal{L}_{\mathcal{C}}$ and $\mathcal{L}$, do not have these problems, but on the contrary, they incorporate these quantum mechanical features explicitly. This is so because they satisfy the conditions listed in section 3. While each state (pure or mixed) induces a measure in the lattice of projections, this has nothing to do with the identification of these measures with classical mixtures. Indeed, any pure state is not dispersion free also and so induces a measure over $\mathcal{L}_{v \mathcal{N}}$. This measure has a radical different nature from that of classical measures; in our approach, improper mixtures are in the same status than pure states and induce measures over $\mathcal{L}_{v \mathcal{N}}$ as well as pure states, but measures and states are not identified. This situation is very different from that of $C M$, in which the measures induced by pure states are trivial.

Our approach -specially $\mathcal{L}_{\mathcal{C}^{-}}$presents itself as a natural logical and algebraic language for the study of topics which involve compound quantum systems such as quantum information processing and decoherence, which concentrate on the study of $\mathcal{C}$ instead of the lattice of projections. In particular, we can map states of the compound system into states of its subsystems at the lattice level, while this cannot be done in the standard $Q L$ approach. Furthermore, $\mathcal{L}_{\mathcal{C}}$ and $\mathcal{L}$ capture the physics behind the fact that we can mix states according to the "mixing principle" of section 2

As discussed in section 5.2, our construction shows a new radical difference with classical mechanics, namely, that of the enlargement of the propositional structure when interactions are involved, a difference which is not clear in the standard $Q L$ approach. 
Moreover, as we showed in section 6.3, $\mathcal{L}_{\mathcal{C}}$ sheds new light into algebraic properties of quantum entanglement via the study of the natural arrows defined between the lattice of the system and its subsystems. The study of these arrows reveals itself as adequate for the of algebraic characterization of entanglement.

Acknowledgements This work was partially supported by the following grants: PIP $\mathrm{N}^{o}$ 6461/05 (CONICET). We wish to thank G. Domenech for careful reading and discussions.

\section{References}

[1] D. D'Espagnat, Conceptual Foundations of Quantum Mechanics (Benjaming, Reading, MA, 1976)

[2] P. Mittelstaedt, The Interpretation of Quantum Mechanics and the Measurement Process (Cambridge Univ. Press, Cambridge, 1998)

[3] K. A. Kirkpatrik, arXiv:quant-ph/0109146 v2 21 Oct 2001

[4] D. D'Espagnat, arXiv:quant-ph0111081 v1 14 Nov 2001

[5] F. Masillo, G. Scolarici and S. Sozzo, arXiv:0901.0795 v1 [quant-ph] 7 Jan 2009

[6] M. Schlosshauer, Decoherence and the Quantum-to-Classical Transition (Springer, New York, 2007)

[7] M. Horodecki, P. Horodecki, and R. Horodecki, in Quantum Information, G. Alber et al., eds. Springer Tracts in Modern Physics Vol. 173 (Springer, Berlin, 2001), p. 151.

[8] G. Birkhoff and J. von Neumann, Annals Math.37 (1936) 823-843.

[9] M. L. Dalla Chiara, R. Giuntini, and R. Greechie, Reasoning in Quantum Theory (Kluwer Acad. Pub., Dordrecht, 2004)

[10] J. M. Jauch, Foundations of Quantum Mechanics (Addison-Wesley, Cambridge, 1968)

[11] C. Piron, Foundations of Quantum Physics (Addison-Wesley, Cambridge, 1976)

[12] D. Aerts, International Journal of Theoretical Physics 39 (2000) 483-496

[13] D. Aerts, J. Math. Phys. 25 (1984) 1434-1441

[14] G. Domenech, F. Holik and C. Massri, J. Math. Phyys. 51 (2010) 052108

[15] B. Mielnik, Commun. math. Phys. 9 (1968) 55-80

[16] B. Mielnik, Commun. math. Phys. 15 (1969) 1-46

[17] B. Mielnik, Commun. math. Phys. 37 (1974) 221-256

[18] J. von Neumann, Mathematical Foundations of Quantum Mechanics, (Princeton University Press, 12th. edition, Princeton, 1996) 
[19] M. Rédei, Quantum Logic in Algebraic Approach (Kluwer Academic Publishers, Dordrecht, 1998)

[20] R. Werner, Phys. Rev. A 40, (1989) 42774281

[21] I. Bengtsson and K. Życzkowski, Geometry of Quantum States: An Intrdoduction to Quantum Entanglement (Cambridge University Press, Cambridge, 2006)

[22] K. Życzkowski, P. Horodecki, A. Sanpera, and M. Lewenstein, Phys. Rev. A 58 (1998) 883

[23] G. Aubrun and S. Szarek, Phys. Rev. A 73, 022109 (2006)

[24] F. Valentine, Convex Sets (McGraw-Hill Book Company, New York-San Francisco-TorontoLondon, 1964) 